\title{
RAPID FOR THE TYPING OF COAGULASE-NEGATIVE STAPHYLOCOCCI IMPLICATED IN CATHETER-RELATED BLOODSTREAM INFECTION
}

\section{Short title: Evaluation of RAPD for typing CoNS}

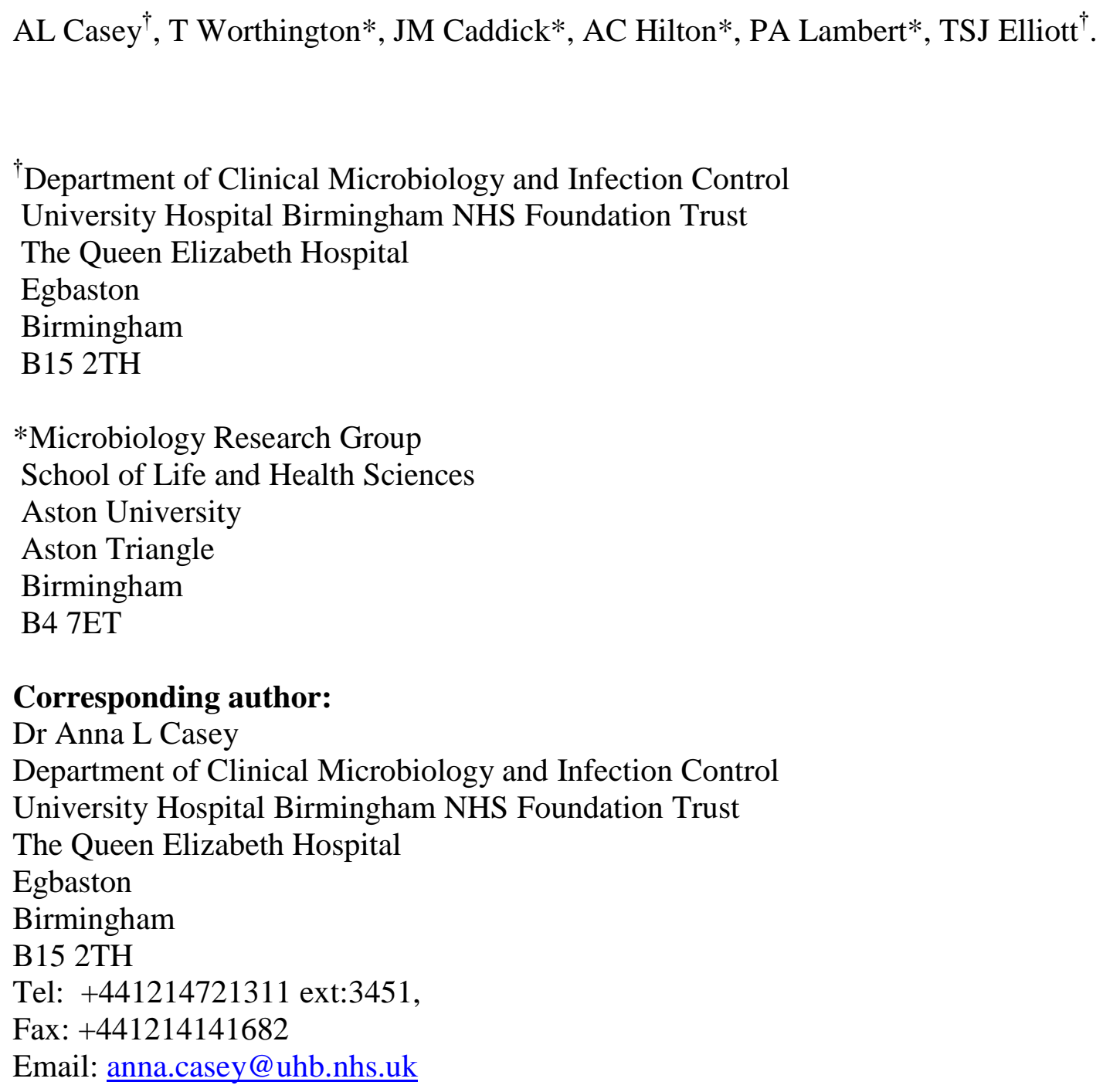




\begin{abstract}
Objectives

A rapid random amplification of polymorphic DNA (RAPD) technique was developed to distinguish between strains of coagulase-negative staphylococci (CoNS) involved in central venous catheter (CVC)-related bloodstream infection. Its performance was compared with that of pulsed-field gel electrophoresis (PFGE).
\end{abstract}

\title{
Methods
}

Patients at the University Hospital Birmingham NHS Foundation Trust, UK who underwent stem cell transplantation and were diagnosed with CVC-related bloodstream infection due to CoNS whilst on the bone marrow transplant unit were studied. Isolates of CoNS were genotyped by PFGE and RAPD, the latter employing a single primer and a simple DNA extraction method.

\section{Results}

Both RAPD and PFGE were highly discriminatory (Simpson's diversity index, 0.96 and 0.99, respectively). Within the 49 isolates obtained from blood cultures of 33 patients, 20 distinct strains were identified by PFGE and 25 by RAPD. Of the 25 strains identified by RAPD, 9 clusters of CoNS contained isolates from multiple patients, suggesting limited nosocomial spread. However, there was no significant association between time of inpatient stay and infection due to any particular strain.

\section{Conclusion}

The RAPD technique presented allows CoNS strains to be genotyped with high discrimination within 4 hours, facilitating real-time epidemiological investigations. In this 
study, no single strain of CoNS was associated with a significant number of CVC-related bloodstream infections.

Key words: Genotyping: random amplification of polymorphic DNA; pulsed-field gel electrophoresis; coagulase-negative staphylococci; central venous catheter-related bloodstream infection. 


\section{Introduction}

Coagulase-negative staphylococci (CoNS) are the most common aetiological agents implicated in nosocomial bloodstream infections associated with central venous catheters (CVC). CoNS accounted for 39.5\% of CVC-related bacteraemias reported to the Nosocomial Infection National Surveillance Scheme between 1997 and 2001 [1]. The increase of CVC-related bacteraemia caused by CoNS is concurrent with advances in modern clinical practice including the use of indwelling medical devices and a rise in the number of immunocompromised patients [2]. Infections associated with CVC in immunocompromised patients undergoing stem cell transplantation (SCT) are associated with high morbidity and mortality rates. Indeed, treatment-induced neutropaenia, the use of immunosuppressive agents and long CVC indwell times place SCT patients at high risk of CVC-related bacteraemia [3].

The association of CoNS with disease and their species diversity has been elucidated by the development of improved methods for their identification and characterisation [4]. Accurate identification and typing of microorganisms is also crucial when undertaking epidemiological investigations. Traditional phenotypic epidemiological markers including antibiogram, biotype, or bacteriophage type are widely used for characterising CoNS in epidemiological studies but in general, they poorly discriminate strains within an individual species [5]. The introduction of genotypic typing methods such as plasmid analysis, DNA hybridization, polymerase chain reaction (PCR)-based typing and pulsed-field gel electrophoresis (PFGE) has improved the characterisation and discrimination of CoNS. Analysis of genetic material is not influenced by environmental factors unlike phenotypic techniques whereby results are dependant on gene expression [6-10]. PFGE is one of the most discriminatory and reproducible typing methods available to genotype CoNS and is 
often regarded as the gold standard. However, despite efforts to reduce the time required to perform this technique, it remains time-consuming and therefore, epidemiological results are often of retrospective value $[6,11-13]$.

The rapid genotypic characterisation method based on the random amplification of polymorphic DNA (RAPD) has been used to genotype CoNS with success in the clinical setting. However the DNA extraction process is often complicated and time consuming [2, 8, 14-16]. Furthermore, the discriminatory power of RAPD has previously been questioned and even the use of multiple primers in the analysis of clonal microorganisms may provide only a limited improvement in discrimination [17-18]. The level of discriminatory power depends heavily on the sequence of the primers employed [19]. In this study, a rapid RAPD protocol employing the use of a single primer and simple DNA extraction technique was developed to characterise and discriminate CoNS strains implicated in CVC-related bloodstream infection in SCT patients at the University Hospital Birmingham NHS Foundation Trust, UK. The performance of the RAPD method was compared with that of PFGE. 


\section{Materials and Methods}

\section{Patients}

Patients who underwent stem cell transplantation (SCT) at the University Hospital Birmingham NHS Foundation Trust, UK were monitored for the presence of CVC-related bloodstream infection due to CoNS based on definitions from the Centre for Disease Control [20]. Only patients who acquired the CVC-related bloodstream infection during their stay on the Bone Marrow Transplant (BMT) Unit were entered into the study.

\section{Bacterial Isolates}

Bacterial isolates obtained from the blood cultures of SCT patients with CVC-related bloodstream infection were identified as CoNS based on conventional laboratory techniques including the catalase test, a staphylococcal latex test kit (Staphytect Plus ${ }^{\circledR}$, Oxoid Ltd, UK) and analytical profile index (API) ID 32 STAPH (BioMerieux, UK).

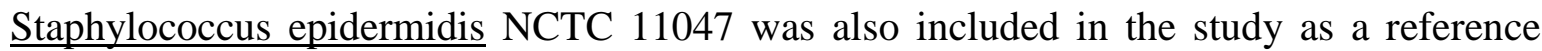
strain.

\section{Genotyping}

\section{PFGE}

Bacterial cells were harvested from an $18 \mathrm{~h}$ culture in brain-heart infusion broth (Oxoid, UK). A wet weight of $20 \mathrm{mg}$ of cells was resuspended in $1 \mathrm{ml}$ NET-100 (0.1 M EDTA (pH 8.0), 0.1 M NaCl, 0.01 M Tris-HCl ( $\mathrm{pH} 8.0)$ ), mixed with an equal volume of molten low melting point chromosomal grade agarose $(0.9 \%(w / v)$ in NET-100; BioRad, UK) and dispensed into a perspex agarose plug casting mould (BioRad, UK). Digestion of the bacterial wall and cellular components was performed as described previously [21-22]. 
Sections $(1 \mathrm{~mm} \times 1 \mathrm{~mm} \times 9 \mathrm{~mm})$ of the agarose plugs containing chromosomal DNA were digested with 50 units of SmaI restriction enzyme (Roche, UK) in $200 \mu$ l sterile distilled water containing $20 \mu \mathrm{l}$ restriction enzyme buffer A (Roche, UK) overnight at $25^{\circ} \mathrm{C}$. The digested sections were subjected to PFGE (CHEF-DR III system, BioRad) using the following parameters: a 1-50 s ramped switch interval with a voltage gradient of $6 \mathrm{~V} \mathrm{~cm}^{-1}$ at an angle of $120^{\circ}$ applied for $24 \mathrm{~h}$ at $10^{\circ} \mathrm{C}$ to a $1 \%(\mathrm{w} / \mathrm{v})$ molecular biology grade agarose gel (BioRad, UK) in $0.5 \times$ TBE buffer (45 mM Tris-Borate, $1 \mathrm{mM}$ EDTA (pH 8.0)). A bacteriophage lambda concatamer ladder DNA size standard (monomer to approximately 21-mer, pulse marker 50-1000 kb, Sigma, UK) was incorporated as a DNA size standard. Following electrophoresis, the gel was stained with $0.5 \mu \mathrm{g} \mathrm{ml}^{-1}$ ethidium bromide for 30 min followed by destaining in distilled water for $1 \mathrm{~h}$.

\section{RAPD}

Isolates were grown on Columbia agar containing $5 \%$ defibrinated horse blood (bioMerieux, UK) and incubated at $37^{\circ} \mathrm{C}$ in air for $18 \mathrm{~h}$. A total of 5 colonies of CoNS were suspended in $1 \mathrm{ml}$ of sterile, double-distilled water and centrifuged for $4 \mathrm{~min}$ at 9000 g. The supernatant was discarded and the step repeated twice after which the cells were resuspended in $1 \mathrm{ml}$ of sterile double distilled water and the absorbance adjusted to 1.7 at $600 \mathrm{~nm}$. $100 \mu \mathrm{l}$ of this suspension was then placed into individual sterile $1.5 \mathrm{ml}$ microcentrifuge tubes and incubated at $95^{\circ} \mathrm{C}$ for $12 \mathrm{~min}$ in a heating block (Techne, UK). A PCR reaction was performed using a 10-mer primer with the sequence; 5'-AGC GTC ACT G-3' (MWG-Biotech AG, Germany) [23]. The reaction was carried out in a $25 \mu 1$ reaction volume containing $18.55 \mu \mathrm{l}$ sterile distilled water, $0.5 \mu \mathrm{l}$ of $10 \mathrm{mM} \mathrm{dNTPs}$ (Promega, UK), $0.25 \mu \mathrm{l}$ of 5 units $\mu^{-1}$ Taq DNA polymerase (Promega, UK), $1.2 \mu \mathrm{l}$ of 10 pM primer, $2 \mu \mathrm{l}$ of DNA template and $2.5 \mu \mathrm{l} 10$ x PCR buffer $\left(\mathrm{pH} 8.3,1.5 \mathrm{MgCl}_{2} \mathrm{mmol} \mathrm{1}^{-}\right.$

${ }^{1}, \mathrm{KCl} 75 \mathrm{mmol} 1^{-1}$ [24]). A no-DNA control was performed by adding $2 \mu \mathrm{l}$ of sterile 
distilled water. The amplification procedure comprised of one cycle for $4.5 \mathrm{~min}$ at $94^{\circ} \mathrm{C}$, followed by 5 low stringency cycles of $30 \mathrm{~s}$ at $94^{\circ} \mathrm{C}, 2 \mathrm{~min}$ at $20^{\circ} \mathrm{C}$ and $2 \mathrm{~min}$ at $72^{\circ} \mathrm{C}$ and 35 high stringency cycles of $30 \mathrm{~s}$ at $94^{\circ} \mathrm{C}, 1 \mathrm{~min}$ at $32^{\circ} \mathrm{C}$ and $2 \mathrm{~min}$ at $72^{\circ} \mathrm{C}$ (PTC-100, MJ Research Inc, Waltham, USA). The amplification was concluded with 5 min at $72^{\circ} \mathrm{C}$ and the reaction products stored at $-20^{\circ} \mathrm{C}$ until required [15]. A $15 \mu$ l volume of each test mixture and $5 \mu \mathrm{l}$ of a $1 \mathrm{~kb}$ DNA molecular mass ladder (Hyperladder IV, Bioline, UK) were then loaded into separate wells in a $2 \%(\mathrm{w} / \mathrm{v})$ agarose gel containing $1 \mu \mathrm{g} \mathrm{ml} \mathrm{m}^{-1}$ of ethidium bromide (Sigma, UK) and electrophoresed at $100 \mathrm{~V}$ for $1 \mathrm{~h}$ in TAE buffer (40 mM Tris, 1 mM EDTA (pH 8.0), 0.1\% glacial acetic acid (Fisher Scientific, UK)).

\section{Visualisation and analysis of RAPD and PFGE profiles}

All electrophoresed gels were visualized with a UV light scanner (UVP Products, UK). RAPD and PFGE fingerprints were analysed by GelCompar (Applied Maths, Belgium). Comparison of the bacterial isolates was carried out by calculation of the Dice correlation coefficient and clustering was performed by the unweighted pair group method of arithmetic averages (UPGMA) in order to construct a dendrogram. For RAPD, only amplicons of $200 \mathrm{bp}$ or larger were analysed and for PFGE, analysis of fragments with a molecular weight of $48.5 \mathrm{~kb}$ or larger only was carried out. The discriminatory power of each typing method was calculated using Simpson's diversity index [25]. 


\section{Results}

\section{Patients}

Thirty-three SCT patients were entered into the study. They had a confirmed clinical and microbiological diagnosis of CVC-related bloodstream infection due to CoNS that was acquired during their stay on the BMT unit. These patients had a total of 37 infectious episodes from which 49 pure cultures of CoNS were obtained from blood samples. Patient demographics, clinical presentation and type of SCT are outlined in table 1.

\section{Bacterial isolates}

One (2\%) out of the 49 isolates of CoNS recovered from the blood culture of a patient with CVC-related bloodstream infection was non-identifiable by the API ID 32 STAPH system. Five different species of CoNS were identified in the remaining 48 isolates: $\underline{\text { S. epidermidis }}$ (23); Staphylococcus haemolyticus (15); $\underline{\text { Staphylococcus hominis (6); Staphylococcus }}$ chromogenes (2) and Staphylococcus xylosus (1).

\section{Genotyping}

\section{PFGE}

Forty-seven out of 49 (96\%) isolates of CoNS implicated in CVC-related bloodstream infection were typeable by PFGE. Macrorestriction fragment analysis of the 23 typeable isolates of $\underline{\mathrm{S}}$. epidermidis produced a range of restriction profiles all of which had 10 to 13 fragments within the 48.5 to $485 \mathrm{~kb}$ range. The 15 isolates of $\underline{\mathrm{S}}$. haemolyticus produced macrorestriction profiles with between 7 and 14 fragments within this molecular weight

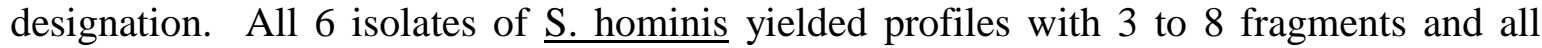

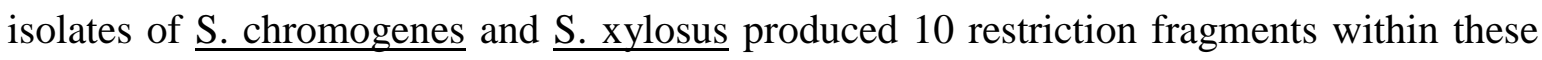
molecular weight limits. The relationship between the isolates of CoNS based on these 
macrorestriction fragment profiles is illustrated in a dendrogram (figure 1). Overall, 37 distinct macrorestriction fragment profiles were identified with a genetic similarity of $38 \%$. PFGE typing of isolates from this patient group resulted in a discriminatory index of 0.99 . Tenover and colleagues' [26] criteria for bacterial strain typing using PFGE state that restriction fragment profiles with between 0 and 3 fragment differences are consistent with a single genetic event and are therefore probably the same strain. Taking this into consideration, 20 different strains of CoNS were characterised during the study when using PFGE and the Tenover criteria (strains A-T, figure 1). At 62\% similarity, 3 main clusters were generated, cluster A comprising primarily of $\underline{S}$. haemolyticus, cluster $\mathrm{B}, \underline{\mathrm{S}}$.

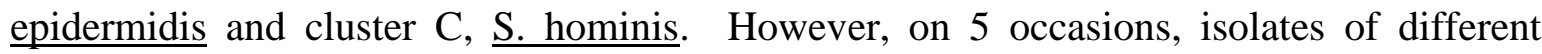
species of CoNS were found within the same strain. Eight out of 11 (73\%) patients (patients 4, 7, 8, 17, 18, 25, 26, and 29) who presented with multiple episodes of CVCrelated bloodstream infection had the same strain of CoNS recovered from at least 2 separate infectious episodes. Conversely, 10 individual strains (strains A, D, I, J, M, N, O, $\mathrm{Q}, \mathrm{R}$ and $\mathrm{T}$ ) contained isolates recovered from different patients.

\section{RAPD}

Forty-seven out of $49(96 \%)$ isolates of CoNS implicated in CVC-related bloodstream infection were typeable by RAPD, the resultant profiles consisting of clear amplicons,

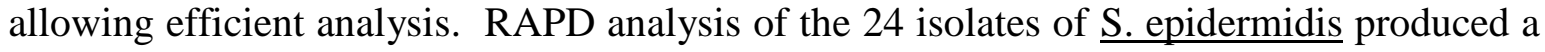
range of profiles, all of which had 3 to 6 amplicons within the 200 to $1500 \mathrm{bp}$ range.

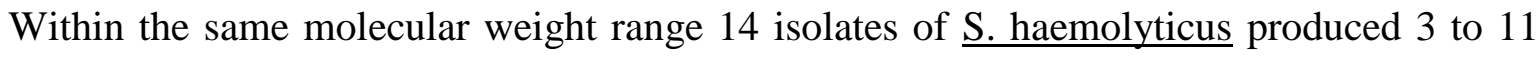
amplicons, 6 to 11 amplicons were achieved from the 6 isolates of $\underline{\mathrm{S} \text {. hominis }}$ and 7 and 5 amplicons from $\underline{S \text {. chromogenes }}$ and $\underline{S \text {. xylosus, }}$, respectively. The relationship between CoNS RAPD profiles was determined by UPGMA dendrogram cluster analysis (figure 2). Twenty-five distinct RAPD types were identified and represented in a cluster at $30 \%$ 
similarity (RAPD types A-Y, figure 2). This RAPD protocol for typing CoNS was highly discriminatory with an index of 0.96 . There are currently no interpretive guidelines for bacterial strain typing using RAPD and since the whole bacterial genome is not sampled using this technique, clonal relationships are difficult to decipher. Strain differentiation when determined using RAPD is therefore often based on single amplicon differences in

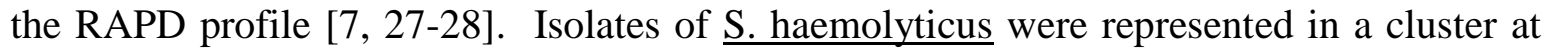

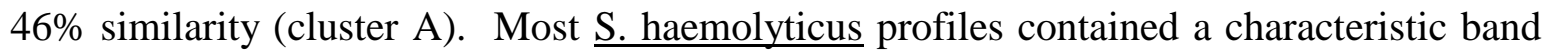
larger than 1000 bp (band pattern A, figure 2) which was absent in other CoNS species. Five out of $6(83 \%)$ isolates of $\underline{\text { S. hominis }}$ were clustered at a similarity of $67 \%$ (cluster B)

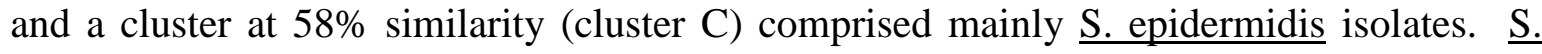
epidermidis profiles were characterised by a 3 band arrangement (440 bp, 510 bp and 760 bp) (band pattern B, figure 2), this pattern was also absent in other species of CoNS. Four RAPD types (A, J, O and P) contained isolates from different species of CoNS (as determined by API ID32 STAPH). When typing using RAPD, 5 out of 11 (45\%) patients (patients 7, 8, 17, 18 and 26) who presented with multiple episodes of CVC-related bloodstream infection had CoNS with identical RAPD profiles isolated from at least 2 separate infectious episodes. Conversely, 9 individual RAPD types (RAPD types A, C, D, $\mathrm{E}, \mathrm{J}, \mathrm{K}, \mathrm{L}, \mathrm{O}$, and S) contained isolates from different patients. Indeed, both RAPD types L and $\mathrm{O}$ contained isolates recovered from 5 different patients. 


\section{Discussion}

The heterogeneic nature of CoNS implicated in nosocomial infection was demonstrated using both PFGE and RAPD. Indeed, genetic heterogeneity was not only demonstrated

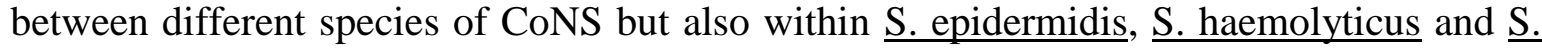
hominis, respectively. Both RAPD and PFGE were highly discriminative with an index greater than 0.9 , which is desirable if the results of a typing scheme are to be interpreted with confidence.[25] The 2 genotypic techniques therefore appear to have the discriminative power to distinguish between species. Indeed, the fact that isolates from different species were found within the genetic strain may reflect the poor specificity of the API system used to speciate the CoNS.

Using RAPD, $45 \%$ of patients who presented with multiple episodes of CVC-related bloodstream infection had the same strain of CoNS recovered from at least 2 separate infectious episodes. This may indicate that each of these patients had a recurrence of their CVC-related bloodstream infection, perhaps due to ineffective antimicrobial therapy and/or bacterial seeding of the blood from the colonised CVC. Thus, genotypic characterisation may provide a useful tool when determining if an intravascular catheter remains a constant source of infection, allowing the clinican to make an informed decision as to whether the source should be removed.

Genotypic analysis of CoNS isolates from SCT patients with CVC-related bloodstream infection illustrated that small clusters of infection may be caused by identical strains perhaps from a common source. However there was no significant association between time of inpatient stay and infection due to any one strain of CoNS. No single persistent strain was identified within the BMT unit during this time period. 
The PFGE technique further sub-divided RAPD types and vice versa, similar to previous findings [29]. It has been demonstrated that if the time capacity and financial resources are available to perform both PFGE and RAPD on a group of bacterial isolates, the combined data result in a higher discriminatory power [30]. Furthermore, the characteristic bands

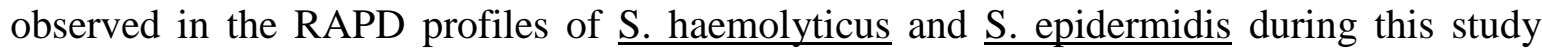
could be sequenced and DNA probes based on these sequences may provide an alternative, more sensitive approach to biotyping in order to speciate CoNS.

It is currently unlikely that the relatively expensive and time-consuming genotypic technique of PFGE will be incorporated routinely into the clinical microbiology laboratory to aid epidemiological investigations. However, the rapid, inexpensive RAPD technique described in this study may provide a potential method whereby strains of CoNS can be rapidly genotyped with high discrimination within 4 hours, thus providing the microbiologist with valuable epidemiological information, for example in an outbreak situation. We have found that, by careful optimisation of the protocol, RAPD generates stable, reproducible results and therefore provides a robust method for CoNS strain characterisation in a clinical setting.

\section{Acknowledgments}

We thank all the staff on the haematology ward and BMT unit, University Hospital Birmingham NHS Foundation Trust, namely, Dr Prem Mahendra and Professor Charles Craddock for allowing us to study their patients and Angela D’Souza, Jane Gray and Elaine Garnett for their help and kindness during the study. 


\section{References}

[1] Coello R, Charlett A, Ward V et al. Device-related sources of bacteraemia in English hospitals-opportunities for the prevention of hospital-acquired bacteraemia. J Hosp Infect 2003; 53(1):46-57.

[2] Nouwen JL, van Belkum A, de Marie S et al. Clonal expansion of Staphylococcus epidermidis strains causing Hickman catheter-related infections in a hemato-oncologic department. J Clin Microbiol 1998; 36(9):2696-702.

[3] Zitella L. Central venous catheter site care for blood and marrow transplant recipients. Clinical Journal of Oncology Nursing 2003; 7(3): 289-98.

[4] Kloos WE, Bannerman TL. Update on clinical significance of coagulase-negative staphylococci. Clin Microbiol Rev 1994; 7(1):117-140.

[5] Lina B, Forey F, Tigaud JD, Fleurette J. Chronic bacteraemia due to Staphylococcus epidermidis after bone marrow transplantation. J Med Microbiol 1995; 42(3): 156-60.

[6] Sloos JH, Dijkshoorn L, Vogel L, van Boven C.P. Performance of phenotypic and genotypic methods to determine the clinical relevance of serial blood isolates of Staphylococcus epidermidis in patients with septicemia. J Clin Microbiol 2000; 38(7):2488-93.

[7] De Mattos EM, Teixeira LA, Alves VM et al. Isolation of methicillin-resistant coagulase-negative staphylococci from patients undergoing continuous ambulatory peritoneal dialysis (CAPD) and comparison of different molecular techniques for discriminating isolates of Staphylococcus epidermidis. Diagn Micr Infec Dis 2003; 45(1): 13-22.

8] Young KA, Power EG, Dryden MS, Phillips I. RAPD typing of clinical isolates of Staphylococcus haemolyticus. Lett Appl Microbiol 1994; 18(2): 86-9. 
[9] Vermont CL, Hartwig NG, Fleer A et al. Persistence of clones of coagulase-negative staphylococci among premature neonates in neonatal intensive care units: two-center study of bacterial genotyping and patient risk factors. J Clin Microbiol 1998; 36(9): 2485-2490.

[10] Schlegel L, Saliba F, Mangeney N, Mathieu D. Pulsed field gel electrophoresis typing of coagulase-negative staphylococci with decreased susceptibility to teicoplanin isolated from an intensive care unit. J Hosp Infect 2001; 49(1): 62-8.

[11] Chang N, Chui L. A standardized protocol for the rapid preparation of bacterial DNA for pulsed-field gel electrophoresis. Diagn Micr Infec Dis 1998; 31(1):275-9.

[12] Matushek, MG, Bonten MJ, Hayden MK. Rapid preparation of bacterial DNA for pulsed-field gel electrophoresis. J Clin Microbiol 1996; 34(10):2598-600.

[13] Leonard RB, Carroll KC. Rapid lysis of gram-positive cocci for pulsed- field gel electrophoresis using achromopeptidase. Diagn Mol Pathol 1997; 6(5):288-91.

[14] Welsh J, McClelland M. PCR-amplified length polymorphisms in tRNA intergenic spacers for categorizing staphylococci. Mol Microbiol 1992; 6(12):1673-80.

[15] Hilton AC, Banks JG, Penn CW. Optimization of RAPD for fingerprinting Salmonella. Lett Appl Microbiol 1997; 24: 243-248.

[16] Bingen E, Barc MC, Brahimi N, Vilmer E, Beaufils F. Randomly amplified polymorphic DNA analysis provides rapid differentiation of methicillin-resistant coagulase-negative staphylococcus bacteraemia isolates in pediatric hospital. J Clin Microbiol 1995; 33(6): 1657-1659.

[17] Raimundo O, Heussler H, Bruhn J.B et al. Molecular epidemiology of coagulasenegative staphylococcal bacteraemia in a newborn intensive care unit. J Hosp Infect 2002; 51(1):33-42. 
[18] Hopkins KL, Hilton AC. Use of multiple primers in RAPD analysis of clonal organisms provides limited improvement in discrimination. Biotechniques 2001; 30(6): 1266-7.

[19] Weller TM. Methicillin-resistant Staphylococcus aureus typing methods: which should be the international standard? J Hosp Infect 2000; 44(3):160-72.

[20] O’Grady NP, Alexander M, Dellinger EP et al. Guidelines for the prevention of IV catheter-related infections. The Hospital Infection Control Practices Advisory Committee, CDC. Pediatrics 2002; 110(5):e51.

[21] Lang S, Livesley MA, Lambert PA, Elliott J, Elliott TSJ. The genomic diversity of coagulase-negative staphylococci associated with nosocomial infections. J Hosp Infect 1999; 43: 187-193.

[22] Lina B, Vandenesch F, Etienne J, Kreiswirth B, Fleurette J. Comparison of coagulasenegative staphylococci by pulsed field gel electrophoresis. FEMS Microbiol Lett 1992; 92, 133-138.

[23] Pereira MSV, Leal NC, Leal TCA et al. Typing of human and bovine Staphylococcus aureus by RAPD-PCR and ribotyping-PCR. Lett Appl Microbiol 2002; 35: 32-36.

[24] Schoettlin W, Neilson KB, Mathur E. Optimisation of PCR using the Opti-prime kit. Strategic Molecular Biology Newsletter 1994; 6: 43-44.

[25] Hunter PR, Gaston MA Numerical Index of the Discriminatory Ability of Typing Systems: an Application of Simpson's Diversity Index. J Clin Microbiol 1998; 26(11): 2465-2466.

[26] Tenover FC, Arbeit RD, Goering RV et al. Interpreting chromosomal DNA restriction patterns produced by pulsed-field gel electrophoresis: criteria for bacterial strain typing. J Clin Microbiol 1995; 33(9):2233-9.

[27] Marsou R, Bes M, Brun Y et al. Molecular techniques open up new vistas for typing of coagulase-negative staphylococci. Pathol. Biol. (Paris) 2001; 49(3):205-15. 
[28] Burnie JP, Naderi-Nasab M, Loudon KW, Matthews RC. An epidemiological study of blood culture isolates of coagulase-negative staphylococci demonstrating hospitalacquired infection. J Clin Microbiol 1997; 35(7):1746-50.

[29] Van Belkum A, Kluytmans J, van Leeuwen W et al. Monitoring persistence of coagulase-negative staphylococci in a haematology department using phenotypic and genotypic strategies. Infect Cont Hosp Ep 1996; 17(10): 660-7.

[30] Van Belkum A, Kluytmans J, van Leeuwen W et al. Multicenter evaluation of arbitrarily primed PCR for typing of Staphylococcus aureus strains. J Clin Microbiol 1995; 33(6):1537-47. 
Table 1. Patient demographics, CVC type, clinical presentation and type of stem cell transplant undergone by patients with CVC-related bloodstream infection

\begin{tabular}{|c|c|}
\hline Mean age (years) & 42 \\
\hline Age range (years) & $18-71$ \\
\hline Male & 20 \\
\hline Female & 13 \\
\hline Catheter type: Hickman line & 26 \\
\hline Peripherally inserted central catheter (PICC) & 4 \\
\hline Short-term non-tunneled CVC & 3 \\
\hline Clinical presentation: Acute lymphoblastic leukaemia & 1 \\
\hline Acute myeloid leukaemia & 6 \\
\hline Chronic myeloid leukaemia & 3 \\
\hline Myelodysplasia & 3 \\
\hline Non-Hodgkins lymphoma & 12 \\
\hline Hodgkins lymphoma & 3 \\
\hline Multiple myeloma & 4 \\
\hline Ewings sarcoma & 1 \\
\hline Stem cell transplant type: Allogeneic sibling PBSC & 5 \\
\hline Mini-allogeneic sibling PBSC & 4 \\
\hline Allogeneic (matched unrelated donor) PBSC & 1 \\
\hline Mini allogeneic (matched unrelated donor) PBSC & 2 \\
\hline Allogeneic (matched unrelated donor) BMT & 5 \\
\hline Autologous PBSC & 16 \\
\hline
\end{tabular}

PBSC (peripheral blood stem cell), BMT (bone marrow transplant) 
Fig. 1. UPGMA dendrogram analysis of PFGE profiles from CoNS isolates implicated in CVCrelated bloodstream infection in SCT patients. Each patient is denoted by a number and in those who experienced multiple episodes of CVC-related bloodstream infection, each episode is represented with an additional letter. Strains identified using the Tenover criteria [26] are labeled $\mathrm{A}$ to $\mathrm{T}$. The day each isolate was recovered during study is recorded. Three major clusters achieved (clusters A-C) are shown, each cluster individually encompassing isolates

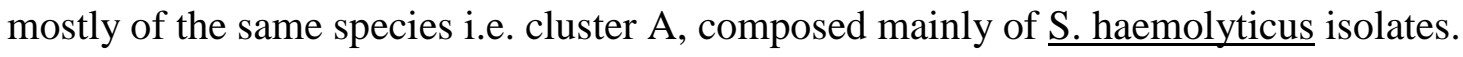

Fig. 2. UPGMA dendrogram analysis of RAPD profiles from CoNS isolates implicated in CVCrelated bloodstream infection in SCT patients. Each patient is denoted by a number and in those who experienced multiple episodes of CVC-related bloodstream infection, each episode is represented with an additional letter. RAPD types, based on a single band difference are labeled A to Y. The day each isolate was recovered during study is recorded. Three major clusters achieved (clusters A-C) are shown, each cluster individually encompassing isolates mostly of the same species i.e. cluster A, composed mainly of $\underline{\mathrm{S} \text {. haemolyticus }}$ isolates. Areas labeled band pattern A and band pattern B illustrate band patterns found almost exclusively in $\underline{\mathrm{S} \text {. haemolyticus }}$

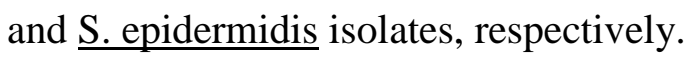


Fig. 1.
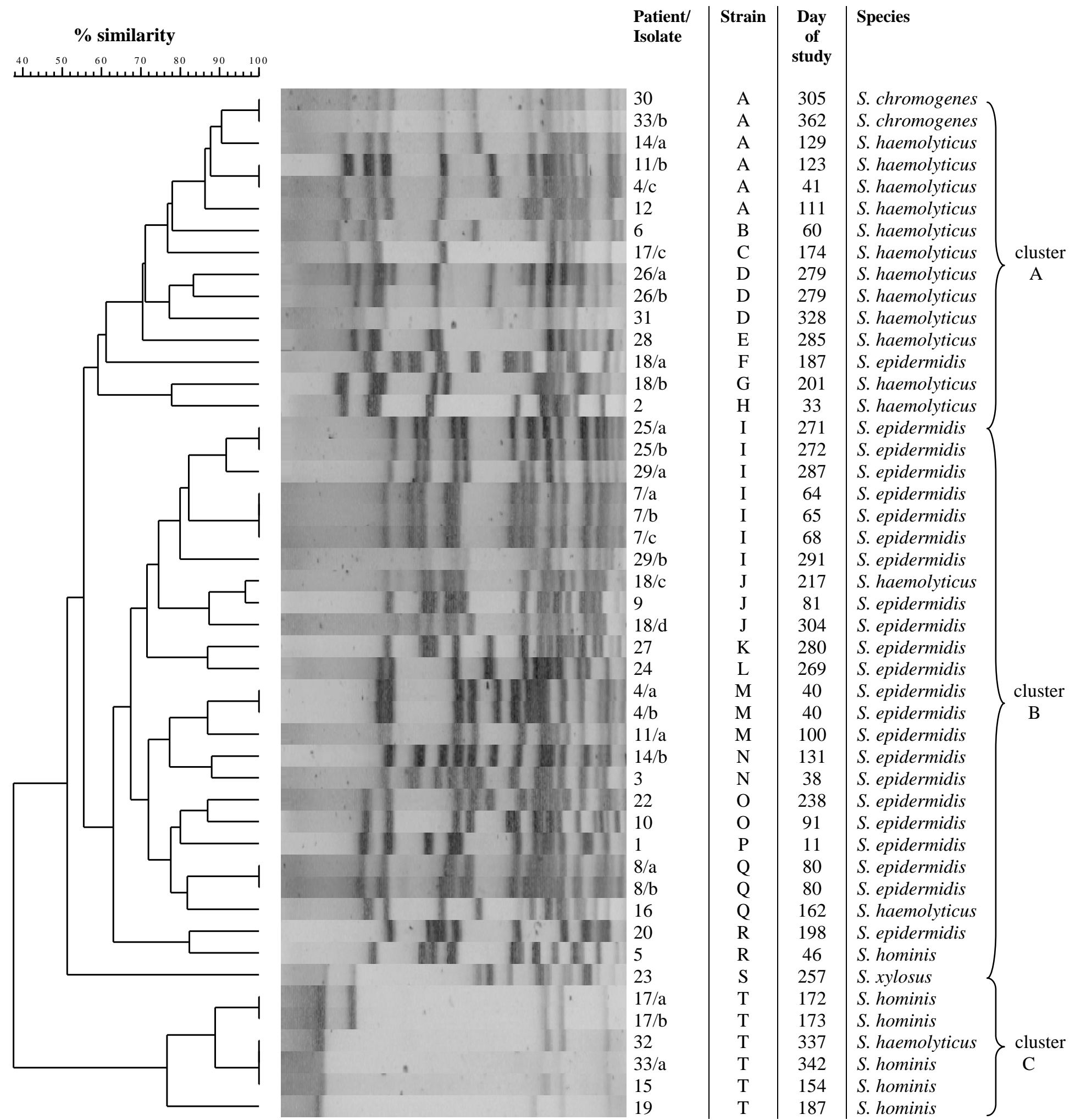
Fig. 2.
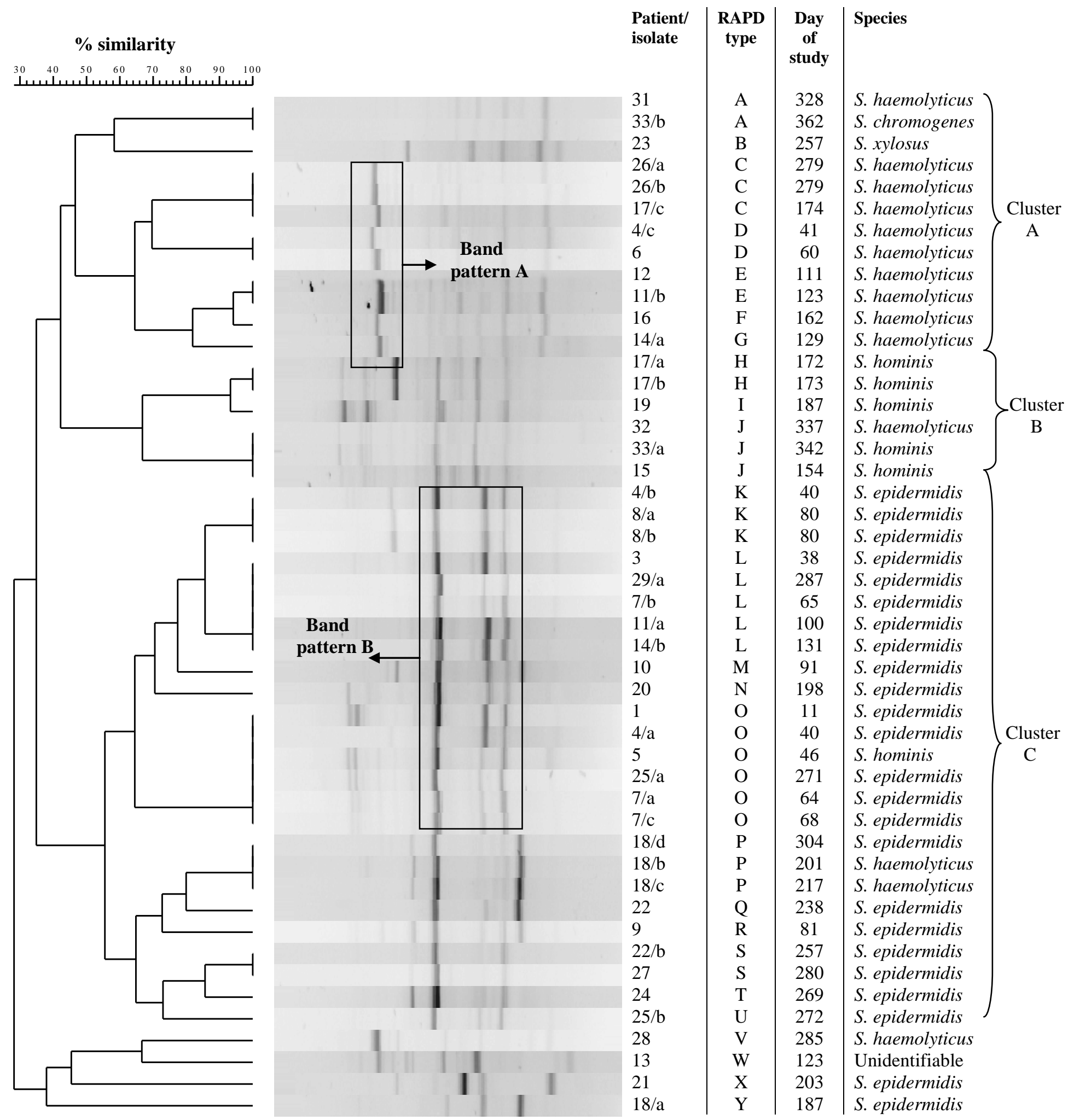
